\title{
Наблюдение локальных и нелокальных электронных квантовых состояний на кремниевой поверхности при комнатной температуре
}

\author{
(C) H.A. Торхов ${ }^{1,2,3}$ \\ ${ }^{1}$ Томский государственный университет, \\ 634050 Томск, Россия \\ ${ }^{2}$ Томский университет систем управления и радиоэлектроники, \\ 634050 Томск, Россия \\ ${ }^{3}$ Севастопольский государственный университет, \\ 299053 Севастополь, Россия \\ E-mail: trkf@mail.ru
}

Поступила в Редакцию 6 мая 2020 г.

В окончательной редакции 24 августа 2020 г.

Принята к публикации 27 августа 2020 г.

\begin{abstract}
Формирование методами атомно-силовой микроскопии на кремниевых поверхностях участков с принудительно измененными одноименными зарядовыми состояниями позволило наблюдать при комнатных температурах когерентные и некогерентные стабильные электронные квантовые объекты. В рамках теории запутанных состояний были определены условия их взаимодействия между собой, объяснены эффекты квантовой экранировки и квантовые интерференционные эффекты между такими объектами.
\end{abstract}

Ключевые слова: кремний, поверхность, когерентные квантовые состояния, квантовая экранировка, квантовая интерференция, запутанные состояния.

DOI: $10.21883 /$ FTP.2021.01.50378.9423

\section{1. Ведение}

Одним из наиболее важных используемых на практике свойств запутанных состояний (entangled states [1,2]) когерентных квантовых систем является то, что при рекогеренции их состояния (редукции, или „схлопывания“" волновой функции) происходит переход замкнутой системы в пространство состояний меньшей размерности, в котором замкнутая квантовая система может иметь свою пространственно-временную метрику, свои энергетические характеристики, свои объекты и свои взаимодействия между ними [3-5]. Такие системы часто проявляют по отношению к внешнему наблюдателю очень необычные свойства, которые по понятным причинам уже не могут быть описаны в рамках классической физики.

Другим важным свойством запутанных состояний когерентных квантовых систем являются интерференционные явления, определяемые законами некогерентной (представленной суммой вероятностей $\Sigma W_{n}$ нахождения системы в различных квантовых состояниях $\psi_{n}$, не имеющих полного набора независимых физических величин) и когерентной (представленной полным набором независимых состояний квантовой системы $\left|\psi_{n}\right\rangle$ ) суперпозиций.

Следствием первой являются смешанные состояния незамкнутой квантовой системы, в определении которых принимают участие только средние значения волновых функций. По этой причине различные некогерентные квантовые состояния не могут интерферировать между собой.

Следствием второй (когерентной суперпозиции) является возможность формирования в замкнутых со- ставных квантовых системах так называемых зацепленных (запутанных) квантовых состояний, а также проявления интерференционных эффектов, играющих важную роль в проектировании криптографических квантовых устройств, сверхбыстродействующих квантовых компьютерах.

Интенсивное изучение запутанных квантовых состояний на фундаментальном уровне позволило в настоящее время ряду фирм („MagiQ Technologies“ (Нью-Йорк), http://www.magiqtech.com; „id Quantique“ (Женева), http://www.idquantique.com/products/overview.htm; NEC (Токио); QinetiQ (Фарнборо, Англия)) осуществить их коммерческое использование в криптографических квантовых устройствах управления и связи [6], сверхбыстродействующих квантовых компьютерах [7]. Для повышения эффективности функционирования электронные квантовые системы подобного типа часто эксплуатируются при криогенных (гелиевых) температурах, что негативно отражается на их массогабаритных и эксплуатационных характеристиках.

Исходя из вышеизложенного исследование эффектов квантовой запутанности при повышенных температурах представляет значительный научно-практический интерес. В связи с этим в работе проводятся исследования эффектов квантовой запутанности электронных состояний на кремниевой поверхности при комнатных температурах $[8,9]$.

\section{2. Объекты исследований и методика экспериментов}

В качестве примера успешного применения зондовых методов исследования квантовых эффектов мож- 
но привести основанные на методах атомно-силовой микроскопии (ACM) баллистическую электронноэмиссионную микроскопию и спектроскопию (Ballistic Electron Emission Microscopy (BEEM) and Spectroscopy (BEES)) [10-12].

Для этих целей в работе использовался высоковакуумный атомно-силовой микроскоп „SOLVER-HV“, предназначенный для высоковакуумных (до $10^{-6}$ Topp) атомно-силовых, магнито-силовых и электрических измерений поверхностей проводящих и непроводящих образцов в широком диапазоне внешних магнитных и электрических полей [13]. В измерениях использовался кремниевый кантилевер HA-HR/W $\mathrm{W}_{2} \mathrm{C}$ с активным конструктивным элементом в виде иглы (далее - зонд) радиусом закругления острия $r \approx 35$ нм и металлизированным покрытием из слоя карбида вольфрама $\mathrm{W}_{2} \mathrm{C}$ с работой выхода $q \varphi_{p}\left(\mathrm{~W}_{2} \mathrm{C}\right)=\Phi_{s m} \approx 4.9$ эВ.

В качестве образцов использовались эпитаксиальные $n-n^{+}-\mathrm{Si}\{111\}$ структуры кремния электронного типа проводимости. Концентрация подвижных носителей электрически заряженной мелкой донорной примеси в эпитаксиальном слое $n-\mathrm{Si}$ толщиной 4 мкм составляла $N_{\mathrm{D}^{+}} \approx 4 \cdot 10^{15} \mathrm{~cm}^{-3}$, в низкоомной подложке $n^{+}-\mathrm{Si}-$ $N_{\mathrm{D}^{+}}>10^{18} \mathrm{~cm}^{-3}$. Для обеспечения хорошего электрического контакта к предметному столику к $n^{+}$-подложке формировался сплавной омический контакт на основе металлизации $\mathrm{Ni} / \mathrm{Au}$.

Объектами исследования являлись локальные участки $l_{\Psi}$ (далее - объекты) на поверхности эпитаксиального $n-\operatorname{Si}\{111\}$ с принудительно измененными зарядовыми состояниями приповерхностной области с использованием атомно-силового микроскопа (ACM) „Solve $\mathrm{HV}^{\text {“ }}$ производства „НТ-МДТ“ (рис. 1, $a$ ). Формирование отрицательного электрического заряда $Q_{\text {ind }}^{-}$объекта происходило через наведенные металлизированным зондом в запрещенной зоне энергий кремниевой поверхности металлоиндуцированные состояния $\Psi_{e}^{(0,+)}$ (рис. $1, b$; аналог металлоиндуцированных состояний в контактах Шоттки [14]) путем подвода в заданную точку поверхности острия проводящей иглы кантилевера, на которую в контактном режиме подавалась необходимая разность потенциалов требуемой полярности $U_{p}>0$ [13]. Исследуемый $i$-й объект, для удобства, будем обозначать символом его состояния $\Psi_{i}$.

При контакте с поверхностью $n$-Si игла кантилевера образует точечный наноконтакт металл-полупроводник (М-П) с барьером Шоттки (БШ) $\mathrm{W}_{2} \mathrm{C} / n-\mathrm{Si}$ (далее - контакт) с эффективной (измеренной) высотой $\varphi_{b m} \approx 0.32$ эВ и показателем идеальности $n \approx 2.05$ и соответствующей областью пространственного заряда (ОПЗ) $w_{c h}$. Истинную высоту барьера контакта металл-полупроводник (М-П) с барьером Шоттки (БШ) можно определить как $\varphi_{b} \approx n \cdot \varphi_{b m} \approx 0.66$ эВ [15].

В используемой электрической схеме включения образца с заземлением предметного столика, согласно энергетической диаграмме (рис. $1, b)$, при $U_{p}>0$ игла кантилевера накапливает отрицательный электрический заряд $Q_{\text {tot }}^{-}<0$ (при $U_{p}<0-$ положительный $Q_{\text {tot }}^{+}>0$ ). Проникая в ОПЗ контакта $\mathrm{W}_{2} \mathrm{C} / n-\mathrm{Si}$ металлоиндуцированные состояния $\Psi_{e}^{(0,+)}$ иглы образуют в запрещенной зоне эпитаксиального $n$-Si при $U_{p}>0$ акцепторные состояния, на которые переходит часть электронов накопленного на кантилевере электрического заряда $Q_{\text {tot }}^{-}$. После отвода кантилевера это приводит к формированию на кремниевой поверхности локализованного участка $l_{\Psi} \approx 0.7$ мкм с электрическим зарядом $Q_{\text {ind }}^{-}[8]$. Локализованные участки с электрическим зарядом $Q_{\text {ind }}^{-}$ сохраняются и при отводе кантилевера. На кантилевере остается часть электрического заряда $Q_{p}^{-}$(рис. $\left.1, b\right)$. Таким образом $Q_{\text {ind }}^{-}+Q_{p}^{-}=Q_{\text {tot }}^{-}$.

Визуализация локального изменения зарядовых состояний на поверхности образца осуществлялась методом зонда Кельвина АСМ по локальному $\Delta \Phi$ изменению электростатического потенциала поверхности $\Delta \varphi(x, y)$. Среднее значение электростатического потенциала исходной (без электрически заряженных участков) области поверхности эпитаксиального $n$-Si составило $E_{\mathrm{F}_{s}}(n-\mathrm{Si}\{111\}) \approx 4.1$ эВ.

Метод зонда Кельвина АСМ основан на измерении контактной разности электростатических потенциалов (КРП) КРП $\equiv \Delta \varphi(x, y)$ (далее - потенциал) между иглой кантилевера и поверхностью:

$$
\Delta \varphi(x, y)=\varphi_{p}-\varphi_{S}(x, y),
$$

где $\varphi_{p}=\Phi_{s m}-$ электростатический потенциал поверхности металлизированного покрытия острия иглы кантилевера ( $p$ - probe), а $\varphi_{S}$ - электростатический потенциал ( $S$ - surface) исследуемой полупроводниковой $q \varphi_{S}=E_{\mathrm{F}_{s}}(s-$ semiconductor $)$ поверхности относительно уровня вакуума $E_{\mathrm{vac}}=0$ [13]. Согласно законам электростатики, отрицательный электрический заряд $Q_{\text {ind }}^{-}$должен вызвать на поверхности $n$-Si увеличение КРП $(\Delta \Phi>0)$, что и наблюдалось в экспериментах. Из выражения (1) несложно определить значение электростатического потенциала $\varphi_{s}$ (работу выхода электронов $\left.\Phi_{S}=q \varphi_{S}\right)$ исследуемой поверхности и энергию $E_{\mathrm{F}_{p}}=-\Phi_{S}=-q \varphi_{S}$ относительно $E_{\mathrm{vac}}=0$.

Одновременно с измерениями рельефа $h(x, y)$ и КРП $\Delta \varphi(x, y)$ регистрировались и изображения фазовых контрастов $\Theta(x, y)$ исследуемых участков. По однородности полученных изображений $\Theta(x, y)$ делались выводы о степени влияния воздействия кантилевера на фазовый состав поверхности. Однородность $\Theta(x, y)$ свидетельствовала в пользу того, что изменение КРП на локальных участках поверхности не связано с изменением фазового состава этих участков. Данное утверждение подтверждается и другим фактом, проявляющимся в том, что на воздухе на локальных участках наблюдается постепенная релаксация КРП $\Delta \Phi$ до фоновых значений КРП свободных областей, что может быть объяснено только растеканием электрического заряда под воздействием атмосферного воздуха. 

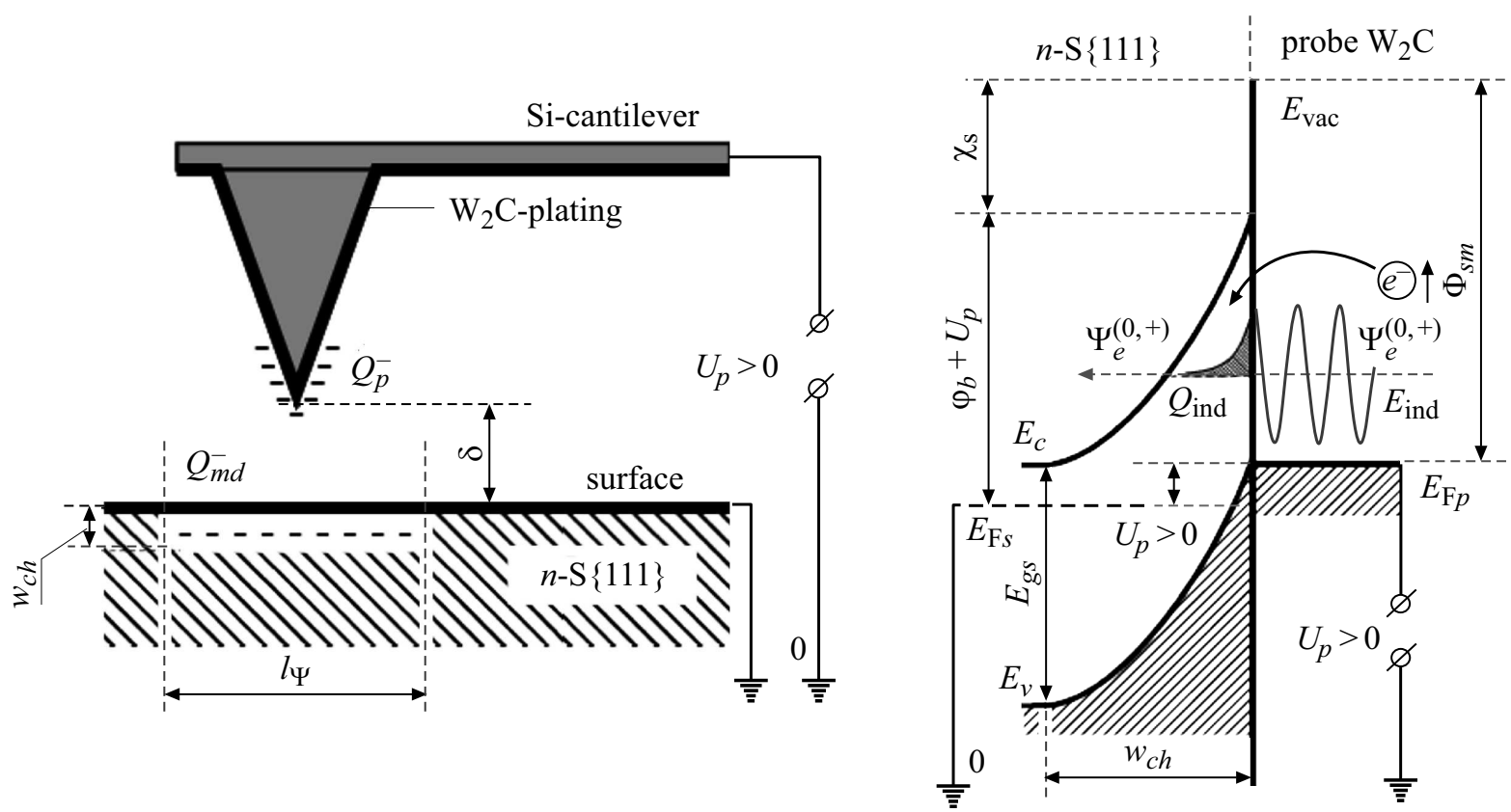

Рис. 1. Схематическое изображение электрического включения квантовой системы зонд-поверхность (cantilever-surface) ( $a$ ) и еe энергетической диаграммы при $\delta=0(b)$. Здесь $E_{\mathrm{vac}}-$ энергия вакуума, $\chi_{S} \approx 4.05$ эВ - электронное сродство $n$-Si, $E_{\mathrm{F}_{s}}-$ уровень Ферми $n-\mathrm{Si}, E_{\mathrm{F}_{p}}$ - уровень Ферми в металлизированной $\mathrm{W}_{2} \mathrm{C}$ игле кантилевера, $\Phi_{s m}$ - работа выхода иглы кантилевера, $E_{\text {ind }}-$ уровень индуцированных состояний, $E_{c}, E_{v}-$ дно зоны проводимости, потолок валентной зоны и $E_{g s}(300 \mathrm{~K}) \approx 1.12$ эВ - ширина запрещенной зоны соответственно.

\section{3. Результаты экспериментов}

Перед началом описания результатов следует отметить, что формирование электрических зарядов на локализованных участках происходило без участия сил зеркальных изображений. На это, в частности, указывает совпадение знаков электрических зарядов на объекте и на игле кантилевера (рис. 1).

Другим необычным обстоятельством является то, что формирование исследуемых объектов не сопровождалось экранирующими эффектами в их классическом понимании. Для таких объектов не выполняется условие нейтральности результирующего электрического заряда в охватывающем объект контуре, превышающем радиус Дебая - основное условие существования дебаевской экранировки $l_{\Psi}$. Согласно рис. $2, a$, исследуемый объект не создает вокруг себя электрического экранирующего эффекта, характерного, например, для контактов Шоттки [16], или $p-n$-переходов [17]. В то же время, несмотря на отсутствие дебаевской экранировки, достаточно сильное $\left(>10^{5} \mathrm{~B} / \mathrm{cm}\right)$ электрическое поле внутри объекта быстро (по „Гауссу“) спадает практически до нуля за его пределами. Получается, что исследуемый объект $\Psi_{1}$, несмотря на отсутствие классической экранировки, практически не взаимодействует с окружающими его электронами проводимости $n$-Si. Очевидно, что наблюдаемый эффект не может быть объяснен в рамках классических представлений.
Не может быть объяснено эффектами экранировки и то, что размер исследуемого объекта (участок $l_{\Phi} \approx 0.7$ мкм, на котором электрическое поле отлично от нуля) значительно превышает протяженность ОПЗ $w_{c h}$ как в объеме $n$ - $\mathrm{Si}$, так и на его поверхности, не зависит ни от величины смещения $U_{p}$ (энергии электронов), ни от времени экспонирования $t$ (концентрации электронов) и в проводимых экспериментах значительно превышает диаметр острия иглы кантилевера $\sim 70$ нм. В общем случае размер локализованного участка $l_{\Psi}$, по-видимому, определяется характером распространения токов растекания и природой системы зонд-поверхность.

Кроме этого, в пределах объекта $l_{\Psi}$ не наблюдается также и какого-либо перераспределения подвижных электронов, которое неминуемо должно было бы происходить в классических системах с подвижными электрическими зарядами одноименной полярности. В случае классической природы объекта горизонтальные сечения трехмерного изображения КРП должны были бы напоминать горизонтальное сечение тора с минимумом в его центре и максимумом по его периметру. Согласно рис. 2, $a$ (см. вставку), сечение КРП объекта представляет собой сплошной круг. При этом профиль вертикального сечения КРП исследуемого объекта достаточно хорошо описывается функцией Гаусса с максимумом в центре объекта. Из этого следует, что электроны объекта стянуты к его центру, а не вытолкнуты на периферию.

Согласно (1), при $E_{\mathrm{vac}}=0$ максимум КРП соответствует максимальной потенциальной энергии объекта 

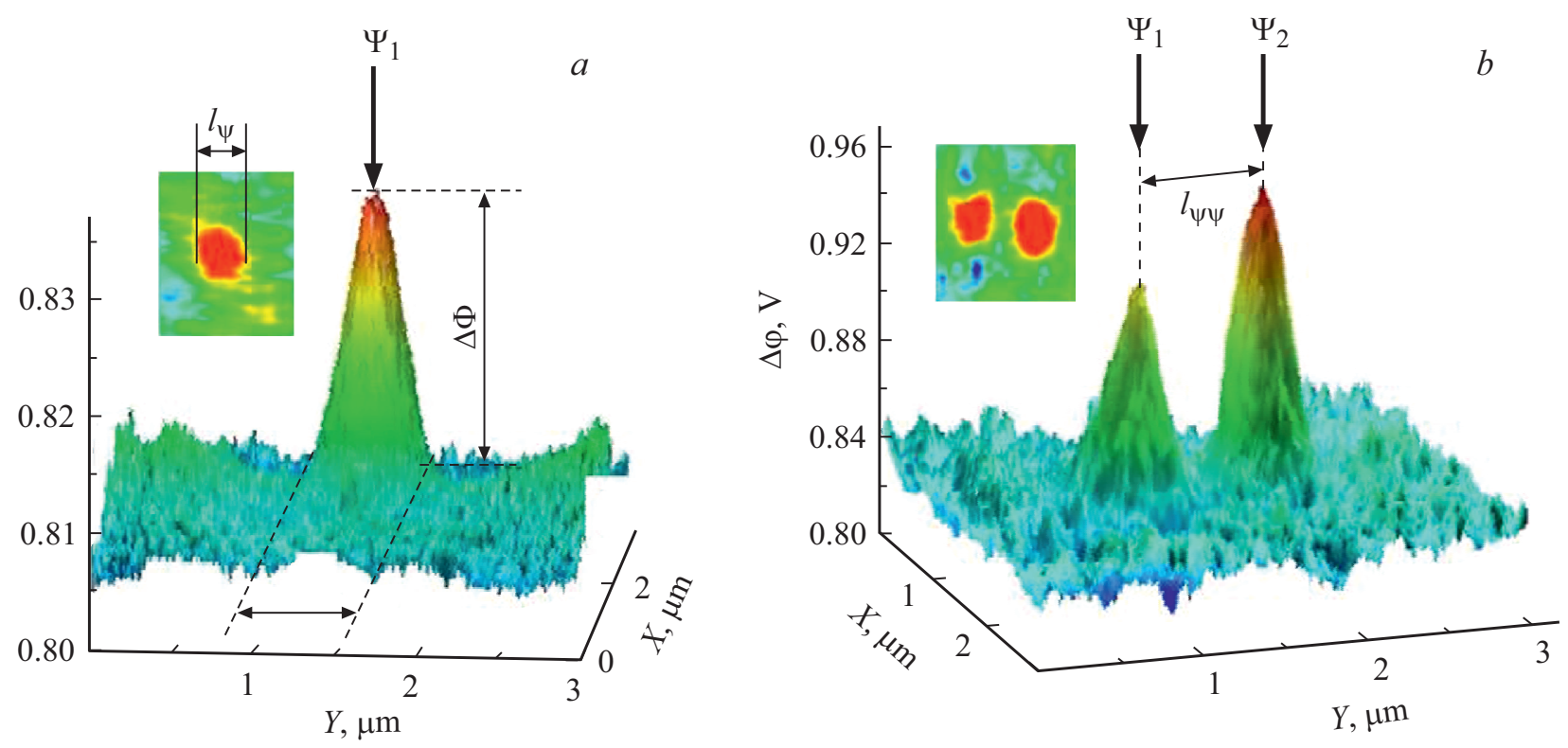

Рис. 2. Трехмерные изображения КРП одиночного квантового объекта $\Psi_{1}(a)$ и двух аналогичных объектов $\Psi_{1}$ и $\Psi_{2}$, находящихся друг от друга на расстоянии $l_{\Psi \Psi} \approx l_{\Psi}(b)$.

$E_{\mathrm{FS} \Psi 1}=-q \varphi_{p}+\Delta \varphi \approx 4.06$ эВ (минимальной работе выхода) относительно среднего значения энергии свободной поверхности $E_{\mathrm{FS}} \approx-4.1$ эВ - максимальной работе выхода (рис. 2,a). Это указывает на то, что исследуемый объект $\Psi_{1}$ находится на поверхности $n-\operatorname{Si}(111)$ в неравновесном термодинамическом состоянии (рис. $1, b)$. Согласно экспериментальным данным, время жизни таких объектов составляет не менее 20 мин, что и позволяет провести их измерение АСМ-методами. Было замечено, что с увеличением размеров объектов наблюдается и увеличение их времен релаксации. Например, для подобных электронных объектов с размерами, превышающими 10 мкм, время релаксации может составлять от единиц до десятков часов.

Требует также специального объяснения и стабильность чистого состояния $\Psi_{1}$ объекта. Напомним, что он образован совокупностью подвижных одноименно заряженных частиц - электронов. Согласно полученным значениям КРП, уровень Ферми в области расположения объекта $l_{\Psi}$ незначительно, на $\sim 35 \mathrm{MB}$, выше уровня Ферми свободной поверхности (рис. 2). С термодинамической точки зрения это означает, что внутренних тепловых флуктуаций в таком объекте должно быть достаточно для переброса электронов в зону проводимости и быстрой их термализации. Понятно, что это должно было бы привести к быстрому исчезновению объекта.

Концентрацию электронов в исследуемых объектах, с одной стороны, можно оценить методами статистической физики по измеряемому положению уровня Ферми $E_{\mathrm{F}_{s}}$ в области объекта $\Psi_{1}$ на уровне $\sim 2.7 \cdot 10^{16} \mathrm{~cm}^{-3}$, что в данном случае практически на порядок превышает концентрацию легирующей примеси. Тем не менее, даже если предположить, что все электроны объекта сосредо- точены на полупроводниковой поверхности, то, исходя из площади объекта (диаметр $\sim 1$ мкм), количество формирующих его электронов должно было бы составлять всего $\approx 650$.

С другой стороны, концентрацию электронов в исследуемых объектах можно оценить через их время фазовой релаксации $\tau$.

В координатном представлении волновую функцию объекта можно записать как

$$
\Psi(x, t)=A(r, t) e^{i \phi(r, t)},
$$

где $r$ - радиус-вектор объекта, $A-$ комплексная амплитуда, $\phi=k x-\varpi t-$ фаза, $k-$ волновой вектор. Так как исследуемые объекты находятся в среде, а не в вакууме, для оценки дополнительного набега фазы $\delta$, необходимого для фазовой релаксации волнового пакета

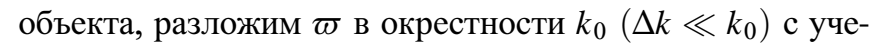
том квадратичного члена разложения:

$$
\begin{gathered}
\varpi(k)=\varpi\left(k_{0}\right)+\Delta k\left[\frac{d \varpi}{d k}\right]_{k=k_{0}}+\frac{(\Delta k)^{2}}{2}\left[\frac{d^{2} \varpi}{d k^{2}}\right]_{k=k_{0}}, \\
\text { тогда } \delta=\frac{(\Delta k)^{2}}{2}\left[\frac{d^{2} \varpi}{d k^{2}}\right]_{k=k_{0}} .
\end{gathered}
$$

Будем полагать, что объект релаксировал, если фаза его волнового пакета за промежуток времени $\tau$ изменилась на $\delta \cdot \tau \approx \pi$. Отсюда можно сделать оценку фазового времени расплывания волнового пакета объекта $\tau$ как

$$
\tau \approx \frac{\pi}{\delta}=\frac{(\Delta x)^{2}}{\frac{d^{2} \varpi}{d k^{2}}},
$$


в котором

$$
\frac{d^{2} \varpi}{d k^{2}}=\hbar \frac{d^{2} E}{d p^{2}}=\frac{\hbar}{m}
$$

Произведя в (4) подстановку знаменателя, сможем оценить по порядку величины $\tau$ как

$$
\tau \approx \frac{(\Delta x)^{2} m}{\hbar}
$$

где $\Delta x=l_{\Psi}, \hbar \approx h / 2 \pi$, масса объекта $m=N \cdot m_{0 e} \cdot m_{e}^{*}$, $m_{0}$ - масса покоящегося электрона, $m_{e}^{*}-$ его эффективная масса в кремнии, $N-$ их количество. Выражение (5) хорошо зарекомендовало себя в оценках по порядку величины времен жизни атомных, нано- и микрообъектов. Используя экспериментально определенное время релаксации $\tau \approx 20$ мин, из (5) можно оценить массу объекта $m$ и количество образующих его электронов как $N>10^{11}$, которое существенно превышает полученный ранее результат. Кроме того, при таком количестве электронов их плотность в объекте была бы аномально большой, что невозможно в рамках обычных представлений.

Если в выражение (5) подставить ранее определенное значение $N \approx 650$, то получится чрезмерно малое значение времени релаксации, $\tau \approx 5$ мкс, недостаточное даже для проведения однократного сканирования участка $3 \times 3$ мкм на $\mathrm{ACM}(\sim 5$ мин $)$.

Понятно, что для исчерпывающего ответа на этот вопрос необходимо проведение дальнейших исследований.

Таким образом, многие наблюдаемые особенности в поведении исследуемых объектов не поддаются объяснению в рамках обычных представлений.

Возможная причина наблюдаемых эффектов, ответственных за декогеренцию электронных состояний в объекте при комнатной температуре, связана, вероятно, с кулоновской блокадой электрон-электронного взаимодействия за счет возникновения на участках с размерами $<5$ нм одиночных изолированных носителей электрических зарядов [18].

При более внимательном рассмотрении было обнаружено, что исследуемый объект, несмотря на относительно большие размеры, представляет собой достаточно простую квантовую систему, образованную совокупностью большого количества находящихся в запутанном состоянии подвижных электронов. Физическая природа такого объекта, как указывалось выше, основана на проникающих из кантилевера в приповерхностную область $n$-Si индуцированных состояниях $\Psi_{e}^{(0,+)}$ (рис. 1, b) [14]. В результате большой плотности таких состояний в пределах $l_{\Psi}$ они могут образовывать подзоны, что и обеспечивает подвижность находящихся в этих состояниях электронов. Несмотря на это, как указывалось выше, перераспределения электронной плотности в пользу ее увеличения по краям объекта под воздействием классических сил электростатического отталкивания не наблюдается.
Поведение исследуемого объекта достаточно просто можно объяснить с точки зрения квантовой физики в рамках теории запутанных состояний тем, что в процессе рекогеренции размерность пространства состояний таких электронов понижается, и они образуют на участке $l_{\Psi}$ несепарабельную достаточно закрытую от внешнего наблюдателя замкнутую систему, характеризующуюся своим чистым квантовым состоянием $\Psi_{i}$ (рис. 1, $a)$ :

$$
\begin{gathered}
\Psi_{i}=\sum_{n=1}^{N} c_{n}^{i} \psi_{n}^{i}, \quad \hat{\rho}=\sum_{n}^{N} W_{n}\left|\psi_{n}\right\rangle\left\langle\psi_{n}\right|, \\
\operatorname{Tr}(\hat{\rho})=\sum_{n}^{N} W_{n}=1,
\end{gathered}
$$

где $\hat{\rho}$ - оператор матрицы плотности, а $W_{n}=\left|c_{n}\right|^{2}-$ вероятность обнаружить систему в одном из возможных состояний $\psi_{n}$. Для чистых состояний замкнутых систем справедливо равенство $\operatorname{Tr}\left(\hat{\rho}^{2}\right)=1$ при $\operatorname{Tr}(\hat{\rho})=1$.

Как было упомянуто выше (см. 1. Введение), состоящая из $N$ подсистем замкнутая квантовая система в чистом состоянии может иметь свою пространственновременную метрику, свои энергетические характеристики, свои объекты и взаимодействия между ними [3-5]. Таким образом, находящиеся в запутанном состоянии электроны замкнутой системы - объекта могут в значительной степени терять для внешнего наблюдателя свои индивидуальные свойства, в том числе, видимо, и в какой-то степени классический характер кулоновского взаимодействия как между собой, так и с ближайшим окружением объекта. Подобную изоляцию от внешнего окружения, находящегося в термодинамически неравновесном состоянии квантового объекта, по аналогии с дебаевской экранировкой будем называть квантовой экранировкой.

Другим полезным обстоятельством является то, что исследуемые квантовые объекты образованы достаточно большим количеством электронов. В результате чего полная внутренняя энергия таких объектов может заметно превышать $k T / q$, что и позволяет наблюдать их при комнатной температуре на фоне тепловых, дробовых и фликкер-шумов.

Результаты дополнительных экспериментов подтверждают утверждение о квантовом состоянии исследуемого объекта, как о чистом квантовом состоянии. Как следует из рис. $2, b$, сформированные независимым образом в непосредственной близости $l_{\Psi \Psi} \approx l_{\Psi}$ друг от друга два одинаковых объекта с некогерентными квантовыми состояниями $\Psi_{1}$ и $\Psi_{2}$, несмотря на одинаковые знаки образующих их электрических зарядов и небольшое расстояние $l_{\Psi \Psi}$, практически никак не взаимодействуют друг с другом, что проявляется на АСМ-изображении в отсутствие между ними общих областей перекрытия. Согласно классическим представлениям, эти объекты должны были бы отталкиваться друг от друга, что на 

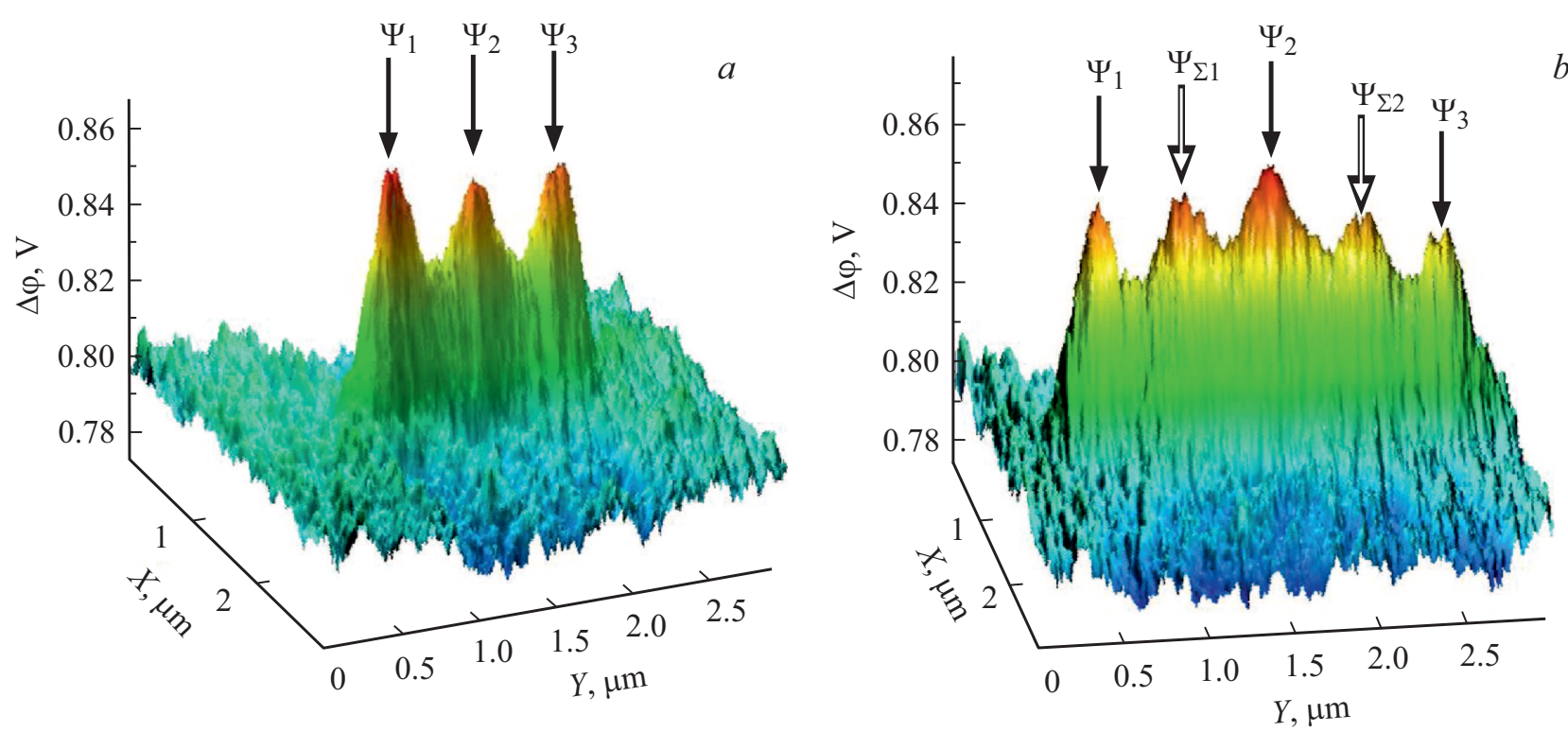

Рис. 3. Трехмерные 3D-изображения КРП трех объектов, находящихся в запутанных квантовых состояниях $\Psi_{1}, \Psi_{2}$ и $\Psi_{3}$ и расположенных на одиночном $l_{\Psi \Psi} \approx l_{\Psi}(a)$ и на двойном расстоянии $l_{\Psi \Psi} \approx 2 l_{\Psi}$ друг от друга с квантовыми интерференционными пиками $\Psi_{\Sigma 1}$ и $\Psi_{\Sigma 2}(b)$.

АСМ-изображении проявлялось бы в изменении формы горизонтальных сечений их КРП - например, в виде направленных в противоположные стороны двух каплеобразных пятен. Такое предположение противоречит полученным результатам, так как их горизонтальные сечения у основания представляют собой практически симметричные круги, свойственные для невзаимодействующих одиночных объектов (см. вставку на рис. $2, b$ ). Эти объекты ведут себя практически полностью независимо друг от друга. Таким образом, квантовые системы с некогерентными состояниями $\Psi_{1}$ и $\Psi_{2}$ можно сдвинуть, а затем разнести на большое расстояние $l_{\Psi \Psi} \rightarrow \infty$, и об их предыстории у них не сохранится никакой информации.

С практической точки зрения это означает то, что находящиеся в некогерентных квантовых состояниях электронные квантовые линии передачи не будут взаимодействовать между собой и обмениваться информацией, даже находясь в непосредственной близости друг от друга.

Таким образом, замкнутость квантовой системы объекта обеспечивает его стабильность во времени, а его достаточная внутренняя энергия - заметность на фоне различного рода шумов.

Отличия в поведении когерентных квантовых систем рассмотрим на примере находящихся в запутанных когерентных квантовых состояниях $\Psi_{1}, \Psi_{2}$ и $\Psi_{3}$ трех идентичных квантовых объектов (рис. $3, a$ ). Согласно рис. $3, a$, расположенные на расстоянии $l_{\Psi \Psi} \approx l_{\Psi}$ объекты не поляризуют вокруг себя среду и не взаимодействуют с окружающими их электронами проводимости $n$-Si, но эффективно взаимодействуют друг с другом, что проявляется на АСМ-изображении в виде областей перекрытия их поверхностных потенциалов в интервалах между $\Psi_{1}$ и $\Psi_{2}$, а также между $\Psi_{2}$ и $\Psi_{3}$. В классическом представлении подобное перекрытие КРП обозначало бы наличие притяжения между объектами, что невозможно для объектов с одинаковым знаком электрических зарядов.

В квантово-механическом же представлении подобное поведение находящихся в среде квантовых систем (объектов) не вызывает противоречий. Взаимодействие объектов, находящихся в когерентных квантовых состояниях, нарушает замкнутость их систем, что приводит к увеличению размерности $N^{*}>N(N-$ размерность пространства состояний каждого из объектов) пространства состояний и возникновению неравенства $\operatorname{Tr}\left(\hat{\rho}^{2}\right)<1$ при $\operatorname{Tr}(\hat{\rho})=1$, которое означает, что электроны объектов в пространстве состояний большей размерности $N^{*}$ переходят в частично запутанные состояния $\Psi \equiv \Psi_{\Sigma}-$ так называемый процесс декогеренции. В этом случае коэффициенты $c_{n}$ будут определять степень запутанности состояний исследуемых объектов, которая и приводит к возникновению между ними корреляционного взаимодействия (рис. $3, a$, области перекрытия $\Psi_{1}-\Psi_{2}$ и $\left.\Psi_{2}-\Psi_{3}\right)$.

Подобного рода взаимодействия между квантовыми когерентными системами могут сопровождаться квантовыми интерференционными эффектами, о которых пойдет речь далее.

Следующий эксперимент направлен на проверку когерентности квантовых состояний $\Psi_{1}, \Psi_{2}$ и $\Psi_{3}$ запутанных объектов (рис. $3, b$ ). Напомним, что в этом эксперименте объекты были не только сформированы одинаковым образом, но и введены в запутанное состояние. Так, увеличение расстояния между объектами в 2 раза $l_{\Psi \Psi} \approx 2 l_{\Psi}$ привело к возникновению интерференционного эффекта, 
проявляющегося в появлении интерференционных (виртуальных) состояний $\Psi_{\Sigma 1}(7)$ и $\Psi_{\Sigma 2}(8)$, расположенных посредине между объектами в состояниях $\Psi_{1}$ и $\Psi_{2}$, а также между объектами в состояниях $\Psi_{2}$ и $\Psi_{3}$ соответственно, что и подтверждает когерентность квантовых состояний этих объектов. При этом взаимодействие между всеми объектами сохраняется:

$$
\begin{aligned}
& \Psi_{\Sigma 1}=\sum_{i=1}^{3} \Psi_{i}, \quad \Psi_{3} \rightarrow 0, \\
& \Psi_{\Sigma 2}=\sum_{i=1}^{3} \Psi_{i}, \quad \Psi_{1} \rightarrow 0,
\end{aligned}
$$

Отметим, что кантилевер контактировал с кремниевой поверхностью только во время формирования объектов $\Psi_{1}, \Psi_{2}$ и $\Psi_{3}$. В пространстве между этими объектами, в том числе и в точках $\Psi_{\Sigma 1}$ и $\Psi_{\Sigma 2}$, взаимодействие кантилевера с поверхностью во время проведения эксперимента не осуществлялось. По всей вероятности, в процессе формирования объектов сохраняются только те индуцированные состояния на полупроводнике, которые были заняты электронами. При отводе кантилевера незанятые электронами индуцированные в полупроводник состояния не сохраняются. Материализацию объектов в виртуальных состояниях $\Psi_{\Sigma 1}$ и $\Psi_{\Sigma 2}$, по всей вероятности, обеспечили электроны проводимости $n-\operatorname{Si}\{111\}$.

В отличие от некогерентных квантовых систем корреляционная связь между запутанными объектами должна сохраняться и в том случае, если их разнести друг от друга на очень большие $l_{\Psi \Psi} \rightarrow \infty$ расстояния. При этом запутанные квантовые объекты всегда будут не только чувствовать присутствие друг друга, но, возможно, и „воспроизводить“ информацию друг о друге в квантовых интерференционных эффектах, как, например, в виде появления виртуальных состояний $\Psi_{\Sigma 1}$ и $\Psi_{\Sigma 2}$. Можно заметить, что состояния $\Psi_{\Sigma 1}$ и $\Psi_{\Sigma 2}$ эквивалентны состояниям $\Psi_{1}, \Psi_{2}$ и $\Psi_{3}$. Иными словами, не зная истории возникновения запутанных состояний, невозможно отличить интерференционные (виртуальные) состояния $\Psi_{\Sigma 1}$ и $\Psi_{\Sigma 2}$ от состояний $\Psi_{1}, \Psi_{2}$ и $\Psi_{3}$ реальных физических объектов.

С практической точки зрения это означает, что находящиеся в когерентных квантовых состояниях квантовые линии передачи могут не только эффективно взаимодействовать между собой, но, вероятно, и формировать интерференционные (виртуальные) копии друг друга. Количество $N-1$ таких копий, по-видимому, должно определяться количеством $N$ участвующих во взаимодействии (в квантовой интерференции) квантовых линий передач.

\section{4. Заключение}

Использование теории запутанных состояний позволило объяснить возможность формирования на кремни- евой поверхности многочастичных электронных квантовых систем - объектов, способных находиться как в когерентных, так и в некогерентных квантовых состояниях. Так, перевод совокупности подвижных электронов в когерентное квантовое состояние позволяет сформировать на кремниевой поверхности при комнатной температуре достаточно стабильные многочастичные квантовые системы, способные находиться по отношению друг к другу как в когерентном, так и в некогерентном квантовых состояниях.

Достаточная замкнутость таких систем обеспечивает их квантовую экранировку от ближайшего окружения и, соответственно, стабильность их состояний во времени, а достаточная внутренняя энергия - хорошую заметность на фоне тепловых, дробовых и фликкер-шумов при комнатной температуре.

Суперпозиция когерентных состояний таких объектов сопровождается проявлением квантовых интерференционных эффектов, проявляющихся в том, что находящиеся в когерентных квантовых состояниях квантовые объекты могут не только эффективно взаимодействовать между собой на любом удалении друг от друга, но и формировать интерференционные (виртуальные) копии друг друга.

\section{Финансирование}

Исследования выполнены при финансовой поддержке Севастопольского государственного университета, идентификатор проекта 42-01-09/90/2020-1.

\section{Конфликт интересов}

Автор заявляет, что у него нет конфликта интересов.

\section{Список литературы}

[1] D. Gross, S.N. Flammia, J.M. Eisert. Phys. Rev. Lett., 102, 190501 (2009).

[2] C.H. Bennett, G. Brassard, C. Crépeau, R. Jozsa, A. Peres, W.K. Wootters. Phys. Rev. Lett., 70 (13), 1895 (1993).

[3] С.И. Доронин. Квант. магия, 1 (1), 1123 (2004).

[4] . S.I. Doronin, E.B. Fel'dman, I.Ya. Guinzbourg, I.I. Maximov. Chem. Phys. Lett., 341, 144 (2001).

[5] S.I. Doronin, E.B. Fel'dman, I.I. Maximov. J. Magn. Reson., 171 (1), 37 (2004).

[6] Г. Стикс. В мире науки (Scientific American), 4, 69 (2005).

[7] T.F. Watson, S.G.J. Philips, E. Kawakami, D.R. Ward, P. Scarlino, M. Veldhorst, D.E. Savage, M.G. Lagally, Mark Friesen, S.N. Coppersmith, M.A. Eriksson, L.M.K. Vandersypen. Nature, 555, 633 (2018).

[8] N. Torkhov. 29th Int. Crimean Conf. „Microwave \& Telecommunication Technology" (CriMiCo'2019), (Sevastopol, Crimea, Russia, Sept. 8-14, 2019), ITM Web of Conf., 30 (13), 08014 (2019).

https://doi.org/10.1051/itmconf/20193008014 
[9] Н.А. Торхов. 10-я Межсунар. научно-практ. конф. по фбизике и технологии наногетероструктурной СВЧ-электроники „Мокеровские чтения“ (Москва, 15-16 мая 2019). В сб. Национального исследовательского ядерного ун-та „МИФИ“ (М., НИЯУ МИФИ, 2019) c. 14.

[10] W.J. Kaiser, L.D. Bell. Phys. Rev. Lett., 60 (14), 1406 (1988).

[11] H. Palm, M. Arbes, M. Schulz. Appl. Phys. A, 56, 1 (1993).

[12] C. Detavernier, R.L. Van Meirhaeghe, R. Donaton, K. Maex, F. Cardon. J. Appl. Phys., 84, 3226 (1998).

[13] В.Л. Миронов. Основы сканирующей зондовой микроскопии (Нижний Новгород, Ин-т физики микроструктур PAH, 2004).

[14] V. Heine. Phys. Rev. A, 138 (6), 1689 (1965).

[15] V.G. Bozhkov, N.A. Torkhov, A.V. Shmargunov. J. Appl. Phys., 109, 073714 (2011).

[16] Н.А. Торхов. ФТП, 52 (10), 1150 (2018).

[17] N.A. Torkhov, V.A. Novikov. Proc. 28th Int. Conf. „Microwave \& Telecommunication Technology" (CriMiCo 2018), (Sevastopol, Russia, Sept. 9-15, 2018) p. 869.

[18] И.С. Бурмистров. Докт. дис. (Ин-т теоретической физики им. Л.Д. Ландау РАН, Черноголовка, 2012).

Редактор Г.А. Оганесян

\section{Observation of local and nonlocal electronic quantum states on silicon surface at room temperature}

N.A. Torkhov ${ }^{1,2,3}$

${ }^{1}$ Tomsk State University, 634050 Tomsk, Russia

${ }^{2}$ Tomsk State University

of Control Systems and Radioelectronics,

634050 Tomsk, Russia

${ }^{3}$ Sevastopol State University,

299053 Sevastopol, Russia

Abstract Formation of forcefully changed similar charge states regions on silicon surfaces by means of atomic force microscopy methods gives an opportunity to observe coherent and non-coherent stable electronic quantum objects at room temperature. Within the entangled states theory the interaction conditions for such objects were defined and quantum shielding and interference effects between them were explained. 